\title{
Topological Transitions from Multipartite Entanglement with Tensor Networks: A Procedure for Sharper and Faster Characterization
}

\author{
Román Orús, ${ }^{1}$ Tzu-Chieh Wei, ${ }^{2}$ Oliver Buerschaper, ${ }^{3,4}$ and Artur García-Saez ${ }^{2}$ \\ ${ }^{1}$ Institute of Physics, Johannes Gutenberg University, 55099 Mainz, Germany \\ ${ }^{2}$ C. N. Yang Institute for Theoretical Physics, State University of New York at Stony Brook, New York 11794-3840, USA \\ ${ }^{3}$ Perimeter Institute for Theoretical Physics, 31 Caroline Street North, Waterloo, Ontario N2L 2Y5, Canada \\ ${ }^{4}$ Dahlem Center for Complex Quantum Systems, Freie Universität Berlin, 14195 Berlin, Germany
}

(Received 18 June 2014; revised manuscript received 13 November 2014; published 19 December 2014)

Topological order in two-dimensional (2D) quantum matter can be determined by the topological contribution to the entanglement Rényi entropies. However, when close to a quantum phase transition, its calculation becomes cumbersome. Here, we show how topological phase transitions in 2D systems can be much better assessed by multipartite entanglement, as measured by the topological geometric entanglement of blocks. Specifically, we present an efficient tensor network algorithm based on projected entangled pair states to compute this quantity for a torus partitioned into cylinders and then use this method to find sharp evidence of topological phase transitions in 2D systems with a string-tension perturbation. When compared to tensor network methods for Rényi entropies, our approach produces almost perfect accuracies close to criticality and, additionally, is orders of magnitude faster. The method can be adapted to deal with any topological state of the system, including minimally entangled ground states. It also allows us to extract the critical exponent of the correlation length and shows that there is no continuous entanglement loss along renormalization group flows in topological phases.

DOI: 10.1103/PhysRevLett.113.257202

PACS numbers: 75.10.Jm, 03.67.Mn, 05.30.Pr

Topological order [1] is a striking property of quantum matter beyond the Landau paradigm and is characterized by an underlying pattern of long-range entanglement. The existence of such a pattern can be detected, quantitatively, by the so-called topological entanglement entropy $S_{\gamma}$ [2]. Other entanglement properties are sensitive to topological order as well [3]. Moreover, under the effect of a local perturbation it is also well known that topological order is generally robust $[4,5]$ and can sustain a finite perturbation. Intuitively, large closed strings and string nets become energetically expensive in a topological phase as a string tension is increased, thus, ultimately favoring a transition towards a topologically trivial phase. A drawback of using entanglement to detect such topological transitions, however, is that it is very difficult to produce sharp numerical evidence. The reason for this is that commonly used methods, such as the calculation of the topological contribution in Rényi entropies [6], suffer from a significant drop in accuracy when close to a quantum critical point [7]; see Fig. 1. Here, we show how multipartite entanglement, in combination with tensor networks, improves accuracies to an almost perfect level and, additionally, is computed orders of magnitude faster than any Rényi entropy.

More specifically, here we use a novel and efficient tensor network method to evaluate the topological contribution to the geometric entanglement (GE) of blocks, which we call $E_{\gamma}$, for a torus partitioned into cylinders. When close to a quantum phase transition, we find that this approach completely outperforms in accuracy and efficiency calculations of Rényi entropies on infinite cylinders with tensor networks [10]. Without describing the technical details, the main result is summarized in Fig. 1. We apply a string tension $g$ (which corresponds to a magnetic field in the Hamiltonian $[12,13]$ ) to certain toric code ground states and compute the topological

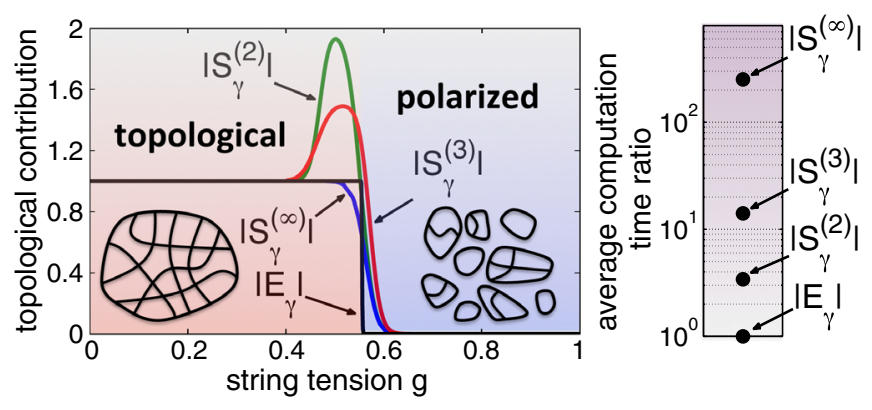

FIG. 1 (color online). Left: phase transition for a topological 2D model with string tension. The black line corresponds to $\left|E_{\gamma}\right|$ as computed in Fig. 4(a) for $n_{b} \rightarrow \infty$ using GE (to be explained later). The rest of the lines correspond to the best achievable calculation by the authors of the topological term $\left|S_{\gamma}^{(n)}\right|$ of the $n$th Rényi entropies $S^{(n)}=(1-n)^{-1} \log \left[\operatorname{tr}\left(\rho^{n}\right)\right]$ of half an infinite cylinder for $n=2,3$ and $n=\infty$ (i.e., the single-copy entanglement [8]), using the methods explained in the Supplemental Material [9]. Compare also to similar calculations with tensor networks in, e.g., Ref. [7]. Typical sizes of string nets populating the ground state for each phase are also represented. Right: average computation time ratio with respect to $\left|E_{\gamma}\right|$, for the different topological contributions. 
contribution of the GE of these "strained" toric code states. Unlike the topological Rényi entropies, the computed $E_{\gamma}$ stays close to -1 throughout the entire topological phase and, as the string tension increases, it sharply drops down to zero at $g^{*} \approx 0.56$ and remains there in the trivial phase. From a mapping to a classical two-dimensional (2D) Ising model [12], we obtain analytically a transition at $g^{*}=\sqrt{1+\sqrt{2}}-1 \approx 0.5537$, in agreement with the above. $E_{\gamma}$ is, thus, an excellent tool to pinpoint topological phase transitions.

Our method uses projected entangled pair states (PEPS) [14]. If the PEPS is topological [15], then a robust $E_{\gamma}$ is extracted via finite-size scaling for large tori. For nontrivial partitions we find $E_{\gamma}$ to depend on the particular superposition of ground states on the torus, in agreement with the behavior of Rényi entropies and entanglement entropy [16]. Our calculations focus mainly on PEPS with a translation invariant representation. This has two main advantages: first, it simplifies the calculations, and second, it corresponds to the type of unique ground state of a topological system that can be found on an infinite plane using, e.g., the infinite projected entangled-pair states (iPEPS) method [17]. Even if such states have a weaker topological contribution on a torus than minimally entangled states (MES), they are much simpler to deal with, and already produce nontrivial topological contributions. In any case, we shall see that our method can be easily extended to PEPS representations that are not invariant under translations, thus, including MES if necessary. Importantly, with this method we also have access to other properties. For instance, in the Supplemental Material [9] we show how to extract the critical exponent $\nu$ and how to see that there is no continuous entanglement loss along renormalization group flows in topological phases [18] together with a fidelity analysis [19].

$G E$ and topological GE.-The geometric entanglement of blocks $[20,21]$ has recently proven useful to assess topological order [22,23]. This multipartite measure has been extensively used in quantum phase transitions [24] and can be measured experimentally, e.g., in NMR [25] and potentially in optical lattice experiments [26]. In contrast to all other entanglement approaches for topological matter, the GE takes into account the multipartite structure of entanglement in quantum many-body states. It amounts to computing the closest product state $|\Phi\rangle$ to a given quantum state $|\Psi\rangle$ in the Hilbert space, where the product state has a separable structure of $n_{b}$ blocks, i.e., $|\Phi\rangle=\prod_{i=1}^{n_{b}}\left|\phi^{[i]}\right\rangle$. It, thus, quantifies the merit of a possible mean-field description of the quantum state. Conveniently, the GE is defined as $E_{G} \equiv-\log |\langle\Phi \mid \Psi\rangle|^{2}$.

One of the latest findings has been that, for renormalization group ( $R G$ ) fixed points such as the toric code and other topological exactly solvable models, the GE of blocks obeys $E_{G}=E_{0}-E_{\gamma}$, with $E_{\gamma}$ a topological contribution (the topological GE) and $E_{0}$ some nonuniversal term [22].
It was observed that $E_{\gamma}=S_{\gamma}$ for the considered models. This constant contribution was shown to be directly connected to the size of the gauge group, which in turn governs topological order in the system. As for $E_{0}$ it was found that $E_{0} \propto n_{b} L$, with $n_{b}$ the number of blocks with a contractible boundary of size $L$. Moreover, under perturbations it was argued that $E_{G}=E_{0}-E_{\gamma}+O\left(L^{-\nu^{\prime}}\right)$ for $L \gg 1$, where again $E_{0} \propto n_{b} L, \nu^{\prime}$ is some exponent, and $E_{\gamma}$ is the (robust) topological term. Recently, the topological GE has also been used to identify minimally entangled ground states, both for Abelian and non-Abelian models [23].

Computing $E_{G}$ and $E_{\gamma}$ from a PEPS.-Our approach to computing the GE of noncontractible blocks $E_{G}$ for large block sizes and its topological contribution $E_{\gamma}$ uses 2D PEPS and 1D matrix product states (MPS). Both PEPS and MPS have been widely discussed in the literature (see, e.g., Ref. [27]). It is worth mentioning that PEPS can describe 2D topological phases naturally, both chiral [28] and nonchiral (or doubled) [15]. For simplicity, here we focus on nonchiral topological order, but a generalization of our method to chiral models is also possible.

As a starting point we assume that a PEPS $|\Psi\rangle$ with (potentially) topological order is given on a torus of $n \times L$ sites; see Fig. 2(a). We call such a state $|\Psi(n, L)\rangle$. This PEPS could be the result of an analytical derivation or have been computed numerically from a Hamiltonian using, e.g., the iPEPS algorithm [17] and later wrapping its tensors around a finite torus.

The goal now is to extract $E_{G}$ and $E_{\gamma}$ from such a PEPS. With this in mind, we partition the torus into $n_{b}$ cylinders of equal width $l=n / n_{b}$. Thus, each cylinder contains $l \times L$

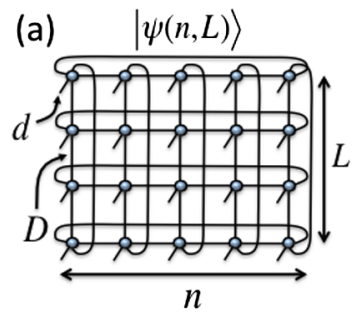

(c)

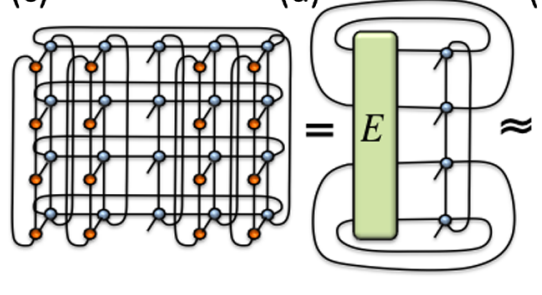

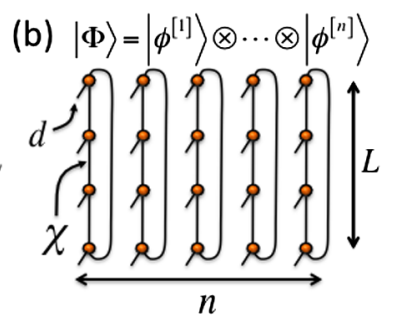

(e)

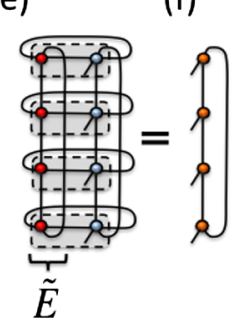

FIG. 2 (color online). (a) PEPS $|\Psi(n, L)\rangle$ wrapped around a torus. (b) Product state $|\Phi\rangle$ of MPS with PBC (cylinders with $l=1$ ). (c) Contraction to compute the optimal state for a given cylinder. (d) Exact result of the contraction in (c) in terms of the environment tensor $E$. (e) Approximation of $E$ by an effective environment $\tilde{E}$ described by an MPO. (f) Resulting optimal MPS for the cylinder in an iteration step (see main text). 
sites; see, e.g., Fig. 2(b). This choice of partition will have a double benefit. First, it will simplify the tensor contractions in the method. Second, it will be sensitive to different ground states on the torus and, hence, to MES.

We focus on the case $l=1$ so that $n_{b}=n$. In this case, one needs to find the closest product state $|\Phi\rangle=\bigotimes_{i=1}^{n_{b}}\left|\phi^{[i]}\right\rangle$ of cylinders of one-site width to $|\Psi(n, L)\rangle$, with $\left|\phi^{[i]}\right\rangle$ the state for cylinder $i$. To do such a calculation efficiently, we further approximate $\left|\phi^{[i]}\right\rangle$ for each cylinder by a MPS of $L$ sites with periodic boundary conditions (PBCs) and bond dimension $\chi$; see Fig. 2(b) [29]. Thus, the original problem is reduced to finding the product state of MPS with PBCs that maximize the overlap with a given PEPS on a torus, which is a well-posed tensor network problem.

In what follows we describe an optimization procedure, well suited for gapped topological phases, to solve this problem. The method assumes a translation invariant PEPS, but it can also be generalized to PEPS without translation symmetry (such as MES).

(1) Assume translation invariance so that cylinders are repeated periodically. While not necessary for a finite system, this assumption simplifies the calculations and also produces good results for translation-invariant PEPS. Here, a two-cylinder unit cell is already sufficient, but bigger unit cells can also be considered.

(2) Fix all tensors in the MPS to some initial (e.g., random) values except for one cylinder and optimize variationally the MPS tensors for that cylinder. The result of this optimization is given by the diagrams in Figs. 2(c) and 2(d). Notice, though, that for a 2D lattice the environment tensor $E$ cannot be computed both exactly and efficiently and, therefore, needs to be approximated.

(3) Compute an effective environment $\tilde{E}$ approximating the exact environment $E$, using some method to approximate contractions of 2D tensor networks. In our case we assume further translation invariance within each cylinder and use the iTEBD method for nonunitary evolutions $[30,31]$, without explicitly implementing the boundary conditions imposed by the torus geometry, and adapted to deal with matrix product operators (MPOs). The specifics are explained in the Supplemental Material [9]. As a result of this approach, an infinite MPO of bond dimension $\chi^{\prime}$ is produced that is then cut at length $L$ and wrapped around a circle with PBC. Such an approximation is particularly accurate for large $L$ and gapped phases, which is precisely the regime of interest to extract $E_{\gamma}$. This finite MPO with PBC describes the effective environment $\tilde{E}$; see Fig. 2(e).

(4) Approximate the optimal MPS for the cylinder as in Fig. 2(f).

(5) Substitute this MPS in all the equivalent cylinders by translation invariance.

(6) Repeat the procedure for the next cylinder in the unit cell.
(7) Iterate until convergence.

The optimal overlap is, thus, evaluated as

$$
\Lambda_{\max }(n, L) \simeq \frac{|\langle\Phi \mid \Psi(n, L)\rangle|}{\sqrt{|\langle\Phi \mid \Phi\rangle||\langle\Psi(n, L) \mid \Psi(n, L)\rangle|}},
$$

with $|\Phi\rangle=\otimes_{i=1}^{n_{b}}\left|\phi^{[i]}\right\rangle$, and $\left|\phi^{[i]}\right\rangle$ the optimal MPS for each cylinder. In this expression, the numerator can be approximated using, e.g., the procedure described in the first section of the Supplemental Material [9] with a computational cost of $O\left(n_{b} \chi^{3} \chi^{\prime 3} D^{5}+L \chi^{\prime 3}\right)$. The norm of $|\Phi\rangle$ is simply the product of the norms of the $n_{b}$ MPS of size $L$ with $\mathrm{PBC}$, which can be evaluated exactly and efficiently in $O\left(L \chi^{5}\right)$ steps (see, e.g., Ref. [27]). The norm of the $n \times L$ PEPS $|\Psi(n, L)\rangle$ can be approximated as in the first section of the Supplemental Material [9] with a computational cost of $O\left(n\left(\chi^{\prime \prime 2} D^{9}+\chi^{\prime \prime 3} D^{6}\right)+L \chi^{\prime \prime 3}\right)$, with $\chi^{\prime \prime}$ the bond dimension of the needed MPO. Finally, the GE is given by $E_{G}(n, L) \equiv-\log _{2} \Lambda_{\max }^{2}(n, L)$.

For an $n \times L$ PEPS on a torus, it is thus possible to approximate $E_{G}(n, L)$ as above. To get the topological contribution, the next step is to perform finite-size scaling with respect to $n$ and $L$. In particular, we have $E_{G}(n \gg 1, L \gg 1) \sim \alpha n L-E_{\gamma}(n, L), \quad$ where $E_{\gamma}(n, L)$ includes both the topological component $E_{\gamma}$ as well as finite-size corrections. We can then fix $n$ and compute $E_{G}(n, L)$ for increasing $L$. Doing a linear fit for large $L$ allows us to extract an approximation to the topological GE by extrapolating the fit down to $L=0$. The larger $n$ is, the more accurate the approximation is. Thus, the value of the topological correction is finally estimated as $E_{\gamma}=\lim _{n, L \rightarrow \infty} E_{\gamma}(n, L)$.

Some remarks are in order. First, accuracy can always be improved by increasing the different bond dimensions or by applying tensor network methods that explicitly take into account PBC rather than iTEBD, or by using larger unit cells (and even completely breaking translation invariance along any direction) in the product state $|\Phi\rangle$. Second, cylinders of larger width $l>1$ can be considered by using a PEPS for an $l$-leg ladder with PBC to approximate the state $\left|\phi^{[i]}\right\rangle$ within each cylinder, or perhaps even a MPS with $\mathrm{PBC}$ and physical dimension $d^{l}$ (with $d$ the physical dimension of a single site). An example of such a calculation is shown in the Supplemental Material [9]. Third, MES can also be studied introducing minor changes in the method. For this, notice that the PEPS representation of an MES is translation invariant except for, e.g., one cylinder where a Wilson loop operator acted. Thus, one chooses $|\Phi\rangle$ as a translation-invariant product state of MPS, except for the Wilson loop cylinder, where a different MPS is chosen. The rest of the method just follows. Fourth, the method relies on single-layer contractions of a $2 \mathrm{D}$ tensor network, which are computed both more efficiently and more accurately than the double-layer contractions in Rényi entropy calculations. This is model independent 

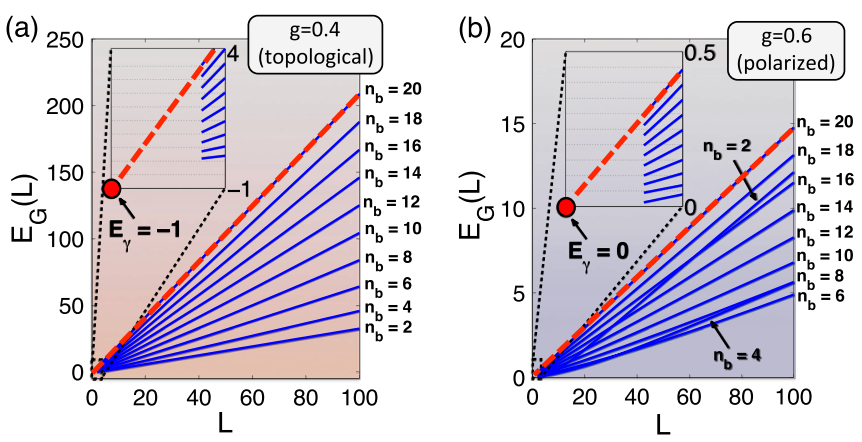

FIG. 3 (color online). $E_{G}(n, L)$ for the toric code with string tension on the square lattice (similar results are also obtained for the honeycomb lattice). (a) Case $g=0.4$. The extrapolation of the linear fit (red dashed line) for, e.g., $n=20$ hits $L=0$ around $\sim-1$ (red dot), as expected in the topological phase. (b) Case $g=0.6$. The same calculation yields $\sim 0$, as expected in the polarized phase.

and explains the overall computational gain from Fig. 1. A more detailed justification is provided in the Supplemental Material [9].

Topological phase transitions from $E_{\gamma}$.-Using the above method, we computed $E_{G}$ and $E_{\gamma}$ for the toric code model [4] with string tension on the square and honeycomb lattices. Details about the PEPS for these models as well as the blocking schemes are given in the Supplemental Material [9]. The string tension $g$ drives the systems towards a phase transition between topological and polarized phases. Using the notation from Ref. [22], we considered perturbations to two nonequivalent ground states $|0,0\rangle$ and $|+,+\rangle$ for the honeycomb lattice, whereas for the square lattice we considered perturbations to the $|0,0\rangle$ state. In the topological phase, these states are the unique ground states of the system on an infinite plane, but on a torus they correspond to a superposition of MES with topological entropy $S_{\gamma}=-1$ for a noncontractible bipartition [16].

Our calculations were done for tori up to $n=100$ and $L=100$. Larger sizes could have easily been considered if necessary. In Fig. 3 we show an example of the scalings with $L$ for different values of $n$ up to $n=20$ for two different string tensions $g=0.4,0.6$ on the square lattice. The linear fit is computed from the last half of $L$ values, which produces robust results. In the plots, the extrapolation of the fit to $L=0$ hits the vertical axis around -1 if $g$ is small, corresponding to the topological phase, and around 0 for large $g$, corresponding to the polarized phase. From the fits we can extract $E_{\gamma}$ as a function of $n=n_{b}$ and $g$, as shown in Figs. 4(a)-4(c) for the three states considered. Remarkably, these plots show very sharp indications of topological phase transitions for all these models for large $n_{b}$. With this approach we also extracted $E_{\gamma}$ for one of the MES of the square lattice toric code on a torus without perturbation. Specifically, we considered the state $\left|\Xi_{0}\right\rangle \equiv 2^{-1 / 2}(|0,0\rangle+$ $|1,0\rangle$ ) (in the notation of Ref. [22]), which has $S_{\gamma}=-2$ for a
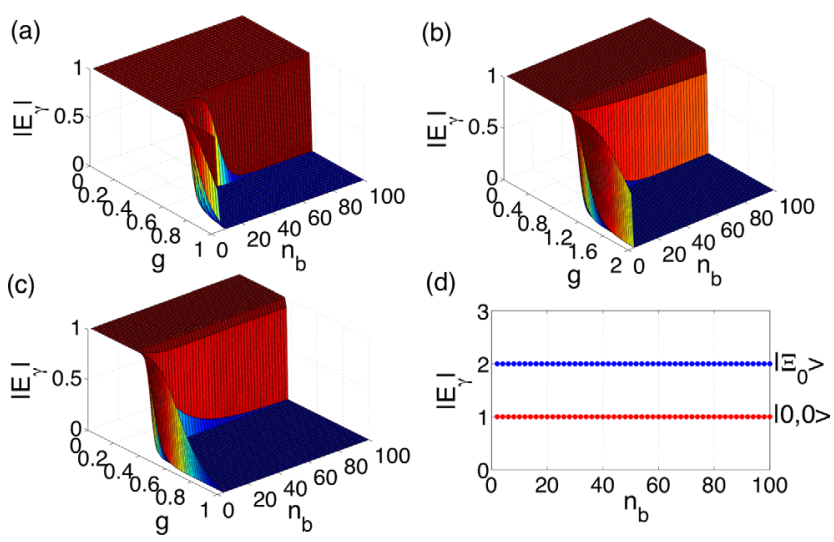

FIG. 4 (color online). Absolute value of $E_{\gamma}$ extrapolated from the scaling with $L$ as in Fig. 3, as a function of the string tension $g$ [for (a)-(c)] and the number of blocks $n_{b}$ [for (a)-(d)]. Plots are for the perturbed (a) $|0,0\rangle$ state on the square lattice, (b) $|0,0\rangle$ state on the honeycomb lattice, and (c) $|+,+\rangle$ state on the honeycomb lattice. Notice that (b) and (c), though being ground states on the same lattice, have different transition points. This is because the string tension $g$ was applied in different bases, hence, corresponding to different physical perturbations. However, we have also checked that when the same perturbation is applied to different ground states on the same lattice the topological phase transition takes place at the same critical point, showing that the transitions in $E_{\gamma}$ do not depend on the specific choice of ground state. (d) Unperturbed toric code on a square lattice for two different ground states: an MES $\left|\Xi_{0}\right\rangle$ and a non-MES $|0,0\rangle$.

nontrivial torus bipartition [16]. Remarkably, we also find $E_{\gamma}=-2$ for this state; see Fig. 4(d).

Conclusions. - We obtained sharp evidence of topological quantum phase transitions for 2D system by calculating $E_{\gamma}$ using a new and efficient tensor network method for nontrivial partitions on a torus. Our method completely outperforms similar tensor network calculations of Rényi entropies for infinite cylinders by being orders of magnitude more accurate and efficient close to criticality [7]. This approach can also be applied to different ground states, including MES, and allows us to extract other nontrivial information about the system (e.g., correlation length critical exponent and lack of continuous entanglement loss along RG flows in topological phases). Our work motivates further research along several directions. For instance, it would be possible to use these tools to study chiral topological order [32], topological critical exponents, and MES. Beyond tensor network methods, it would be interesting to study how to compute $E_{\gamma}$ using quantum Monte Carlo calculations and compare the accuracy and efficiency to that of 2-Rényi entropy calculations.

Discussions with B. Bauer, F. Pollmann, A. Sanpera, and G. Vidal are acknowledged. T.-C.W. and A.G.-S. acknowledge the support by the National Science Foundation under Grants No. PHY 1314748 and No. PHY 1333903. This research was supported in part by Perimeter Institute for Theoretical Physics. Research at Perimeter Institute is 
supported by the Government of Canada through Industry Canada and by the Province of Ontario through the Ministry of Economic Development and Innovation. O. B. acknowledges funding from the ERC (TAQ).

[1] X.-G. Wen, Quantum Field Theory of Many-Body Systems (Oxford University Press, New York, 2004).

[2] A. Kitaev and J. Preskill, Phys. Rev. Lett. 96, 110404 (2006); M. Levin and X.-G. Wen, ibid. 96, 110405 (2006).

[3] S. Iblisdir, D. Perez-Garcia, M. Aguado, and J. Pachos, Phys. Rev. B 79, 134303 (2009); Y. A. Lee and G. Vidal, Phys. Rev. A 88, 042318 (2013); C. Castelnovo, Phys. Rev. A 88, 042319 (2013).

[4] A. Y. Kitaev, Ann. Phys. (Amsterdam) 303, 2 (2003).

[5] A. Hamma and D. A. Lidar, Phys. Rev. Lett. 100, 030502 (2008); S. Trebst, P. Werner, M. Troyer, K. Shtengel, and C. Nayak, Phys. Rev. Lett. 98, 070602 (2007); I. S. Tupitsyn, A. Kitaev, N. V. Prokofev, and P. C. E. Stamp, Phys. Rev. B 82, 085114 (2010); J. Vidal, S. Dusuel, and K. P. Schmidt, Phys. Rev. B 79, 033109 (2009); J. Vidal, R. Thomale, K. P. Schmidt, and S. Dusuel, Phys. Rev. B 80, 081104(R) (2009); J. Yu, S.-P. Kou, and X.-G. Wen, Europhys. Lett. 84, 17004 (2008); X.-G. Wen, Phys. Rev. Lett. 90, 016803 (2003); S. Dusuel, M. Kamfor, R. Orus, K. P. Schmidt, and J. Vidal, Phys. Rev. Lett. 106, 107203 (2011); L. Tagliacozzo and G. Vidal, Phys. Rev. B 83, 115127 (2011); M. D. Schulz, S. Dusuel, R. Orús, J. Vidal, and K. Phillip Schmidt, New J. Phys. 14, 025005 (2012); H. He, H. Moradi, and X.-G. Wen, Phys. Rev. B 90, 205114 (2014); S. Bravyi, M. Hastings, and S. Michalakis, J. Math. Phys. (N.Y.) 51, 093512 (2010); S. Michalakis and M. Zwolak, Commun. Math. Phys. 322, 277 (2013).

[6] S. T. Flammia, A. Hamma, T. L. Hughes, and X. G. Wen, Phys. Rev. Lett. 103, 261601 (2009).

[7] S. V. Isakov, M. B. Hastings, and R. G. Melko, Nat. Phys. 7, 772 (2011); X. Chen, B. Zeng, Z. C. Gu, I. L. Chuang, and X. G. Wen, Phys. Rev. B 82, 165119 (2010); S. Morampudi, C. von Keyserlingk, and F. Pollmann, Phys. Rev. B 90, 035117 (2014); J.-M. Stéphan, G. Misguich, and V. Pasquier, J. Stat. Mech. (2012) P02003.

[8] J. Eisert and M. Cramer, Phys. Rev. A 72, 042112 (2005); R. Orús, J. I. Latorre, J. Eisert, and M. Cramer, Phys. Rev. A 73, 060303(R) (2006).

[9] See Supplemental Material at http://link.aps.org/ supplemental/10.1103/PhysRevLett.113.257202 for a detailed description of the Tensor Network algorithms used in this work.

[10] See, e.g., Ref. [11], as well as the Supplemental Material [9] for the specific algorithms implemented to obtain the data in Fig. 1.

[11] J. I. Cirac, D. Poilblanc, N. Schuch, and F. Verstraete, Phys. Rev. B 83, 245134 (2011).

[12] C. Castelnovo and C. Chamon, Phys. Rev. B 77, 054433 (2008).

[13] S. Trebst, P. Werner, M. Troyer, K. Shtengel, and C. Nayak, Phys. Rev. Lett. 98, 070602 (2007); N. Schuch, D. Poilblanc, J. I. Cirac, and D. Pérez-García, Phys. Rev. Lett. 111, 090501 (2013).
[14] F. Verstraete, J. I. Cirac, and J. I. Latorre, Phys. Rev. A 79, 032316 (2009).

[15] F. Verstraete and J. I. Cirac, Phys. Rev. A 70, 060302(R) (2004); F. Verstraete, M. M. Wolf, D. Perez-García, and J. I. Cirac, Phys. Rev. Lett. 96, 220601 (2006); O. Buerschaper, M. Aguado, and G. Vidal, Phys. Rev. B 79, 085119 (2009); Z.-C. Gu, M. Levin, B. Swingle, and X. G. Wen, Phys. Rev. B 79, 085118 (2009); R. Orús, Ann. Phys. (Amsterdam) 349, 117 (2014).

[16] S. Dong, E. Fradkin, R. G Leigh, and S. Nowling, J. High Energy Phys. 05 (2008) 016; Y. Zhang, T. Grover, A. Turner, M. Oshikawa, and A. Vishwanath, Phys. Rev. B 85, 235151 (2012).

[17] J. Jordan, R. Orus, G. Vidal, F. Verstraete, and J. I. Cirac, Phys. Rev. Lett. 101, 250602 (2008); R. Orús and G. Vidal, Phys. Rev. B 80, 094403 (2009).

[18] B. Swingle, arXiv:1307.8117; S. Santra et al., arXiv:1310.6490; J. I. Latorre, C. A. Lutken, E. Rico, and G. Vidal, Phys. Rev. A 71, 034301 (2005); R. Orús, Phys. Rev. A 71, 052327 (2005); 73, 019904 (2006); A. B. Zamolodchikov, JETP Lett. 43, 730 (1986); I. R. Klebanov, S. S. Pufu, and B. R. Safdi, arXiv:1105.4598.

[19] P. Zanardi and N. Paunković, Phys. Rev. E 74, 031123 (2006); H.-Q. Zhou and J. P. Barjaktarevic, arXiv:cond-mat/ 0701608; H.-Q. Zhou, J.-H. Zhao, and B. Li, arXiv:0704.2940; H.-Q. Zhou, arXiv:0704.2945; H.-Q. Zhou, R. Orús, and G. Vidal, Phys. Rev. Lett. 100, 080601 (2008).

[20] T.-C. Wei and P. M. Goldbart, Phys. Rev. A 68, 042307 (2003).

[21] A. Botero and B. Reznik, arXiv:0708.3391; R. Orús, Phys. Rev. Lett. 100, 130502 (2008).

[22] R. Orús, T.-C. Wei, O. Buerschaper, and M. Van den Nest, New J. Phys. 16, 013015 (2014).

[23] O. Buerschaper, A. García-Saez, R. Orús, and T.-C. Wei, J. Stat. Mech. (2014) P11009.

[24] R. Orús, Phys. Rev. A 78, 062332 (2008); T.-C. Wei, ibid. 81, 062313 (2010); T.-C. Wei, D. Das, S. Mukhopadyay, S. Vishveshwara, and P. M. Goldbart, Phys. Rev. A 71, 060305 (R) (2005); R. Orús, S. Dusuel, and J. Vidal, Phys. Rev. Lett. 101, 025701 (2008).

[25] J. Zhang, T.-C. Wei, and R. Laflamme, Phys. Rev. Lett. 107, 010501 (2011).

[26] A. J. Daley, H. Pichler, J. Schachenmayer, and P. Zoller, Phys. Rev. Lett. 109, 020505 (2012).

[27] R. Orús, Ann. Phys. (Amsterdam) 349, 117 (2014); Eur. Phys. J. B 87, 280 (2014); J. I. Cirac and F. Verstraete, J. Phys. A 42, 504004 (2009); F. Verstratete, J. I. Cirac, and V. Murg, Adv. Phys. 57, 143 (2008); R. Augusiak, F. M. Cucchietti, and M. Lewenstein, in Modern Theories of Many-Particle Systems in Condensed Matter Physics, Lect. Notes Phys. (2012), Vol. 843, p. 245; U. Schollwöck, Rev. Mod. Phys. 77, 259 (2005); U. Schollwöck, Ann. Phys. (Amsterdam) 326, 96 (2011).

[28] J. Dubail and N. Read, arXiv:1307.7726; T. B. Wahl, H. H. Tu, N. Schuch, and J. I. Cirac, Phys. Rev. Lett. 111, 236805 (2013).

[29] This is indeed justified, as we show in the Supplemental Material [9].

[30] G. Vidal, Phys. Rev. Lett. 98, 070201 (2007).

[31] R. Orús and G. Vidal, Phys. Rev. B 78, 155117 (2008).

[32] M. P. Zaletel, R. S. K. Mong, and F. Pollmann, Phys. Rev. Lett. 110, 236801 (2013). 